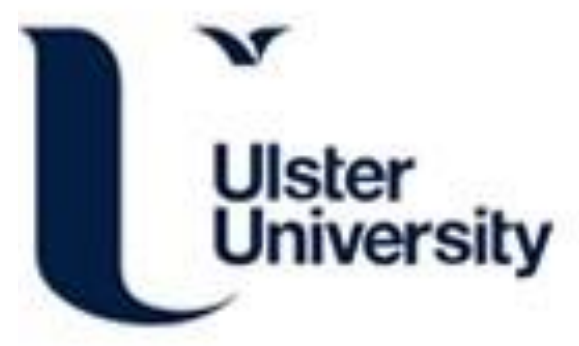

\title{
Fall Detection Through Thermal Vision Sensing
}

Rafferty, J., Synnott, J., Nugent, CD., Morrison, G., \& Tamburini, E. (2016). Fall Detection Through Thermal Vision Sensing. In Ubiquitous Computing and Ambient Intelligence : IWAAL 2016, AmIHEALTH 2016, UCAmI 2016: Ubiquitous Computing and Ambient Intelligence (pp. 84-90). (Lecture Notes in Computer Science). Springer. https://doi.org/10.1007/978-3-319-48799-1_10

Link to publication record in Ulster University Research Portal

\section{Published in:}

Ubiquitous Computing and Ambient Intelligence

\section{Publication Status:}

Published (in print/issue): 01/12/2016

\section{DOI:}

10.1007/978-3-319-48799-1_10

\section{Document Version}

Author Accepted version

\section{General rights}

Copyright for the publications made accessible via Ulster University's Research Portal is retained by the author(s) and / or other copyright owners and it is a condition of accessing these publications that users recognise and abide by the legal requirements associated with these rights.

\section{Take down policy}

The Research Portal is Ulster University's institutional repository that provides access to Ulster's research outputs. Every effort has been made to ensure that content in the Research Portal does not infringe any person's rights, or applicable UK laws. If you discover content in the Research Portal that you believe breaches copyright or violates any law, please contact pure-support@ulster.ac.uk. 


\title{
Fall detection through thermal vision sensing
}

\author{
Joseph Rafferty ${ }^{1}$, Jonathan Synnott ${ }^{1}$, Chris Nugent ${ }^{1}$, Gareth Morrison ${ }^{2}$ and Elena \\ Tamburini ${ }^{3}$ \\ ${ }^{1}$ School of Computing and Mathematics, University of Ulster, Northern Ireland, UK \\ $\{j$.rafferty,j.synnott, cd.nugent \} @ulster.ac.uk \\ ${ }^{2}$ Lava Group 17 Falcon Road, Belfast, UK \\ g.morrisone thelavagroup.co.uk \\ ${ }^{3}$ I+ S.r.l, Piazza Puccini 26, Florence, Italy \\ e.tamburini@i-piu.it
}

\begin{abstract}
Accidental falls can cause serious injury to at risk individuals. This is especially true in the elderly community where falls are the leading cause of hospitalization, injury-related deaths and loss of independence. Detecting and rapidly responding to falls has shown to reduce the long-term impact of and risks associated with falls. A number of real time fall detection solutions exist, however, these have some deficiencies relating to privacy, maintenance, and correct usage. This study introduces a novel fall detection approach that aims to address some of these deficiencies through use of computer vision processes and ceiling mounted thermal vision sensors. A preliminary evaluation has been performed on this process showing promising results, with an accuracy of $68 \%$, however, highlighting a number of issues related to false positives. Future work will improve this approach and provide extended evaluation.
\end{abstract}

Keywords: Fall detection, Assistive technologies, Computer vision, Sensors, Thermal vision.

\section{Introduction}

Accidental falls can cause serious injury to at risk individuals, such as the elderly and those with bone disease. Falls can cause a variety of injuries including broken bones and significant bruising. In particular, broken bones can represent a significant risk to the immediate health of an individual and can negatively affect their long term Quality of Life (QOL) $[1,2]$. This is especially true in elderly communities where falls are the leading cause of hospitalization, injury-related deaths and loss of independence [1-4]. Fall prevention may not be realistically feasible in all cases [5, 6], however, detection of falls and rapidly responding to them has shown to be highly beneficial. Rapid response addresses injury earlier and can help prevent fall-related death and can increase the long-term QOL of elderly individuals who suffered a fall $[4,7,8]$.

A number of solutions currently exist to provide real-time fall detection and alerting. These solutions have shown promising results. They have, however, some deficiencies related to correct usage, privacy and maintenance. In order to address some of these deficiencies, this study investigates the feasibility of a novel approach to fall detection that is based upon thermal sensing and computer vision.

The remaining Sections of this paper are arranged as follows; Section 2 provides a summary of related work; Section 3 introduces the novel approach used within th is study; Section 4 provides an evaluation of the approach and, finally, Section 5 concludes the paper with a reflection on the utility of the approach and future work. 


\section{Related work}

A number of related works exist within the domain of fall detection [9-12]. These are roughly separated into two classes of approach as dictated by their operational model. These classes are Wearable-sens or based and environmental based.

Wearable-sensorbased approaches typically use signals from sensors to detect falls through classifying signals [13]. These sensors include accelerometers, gyroscopes and barometers [14]. These approaches may use dedicated fall detection devices, such as those in [15] or use smartphone based apps [16, 17].

Wearable-sensors have shown extremely promising results with accuracy reaching over $90 \%$ [8], however, have a disadvantage where they need to be worn to function. This represents a problem for use within the elderly population where cognitive impairment may lead to these wearable devices not being used correctly [9-12]. In addition, wearable sensors need to have their power source maintained by a recharging process or battery replacement, representing an additional barrier to adoption.

Environmental approaches rely upon sensors deployed in a location of interest. A range of sensors have been used to in this approach, including acoustic sensors [18], visual/infrared vision cameras $[10,19,20]$, vibration detection [21], positional/pressure tracking [22-24] and thermal vision [25].

Acoustic sensors detect noises which may represent falls. This approach has issues related to noisy environments where its accuracy and utility may be diminished. Visual/infrared cameras show promise, however, represent a potential violation of privacy as they transmit/record highly detailed imagery of an environment. Depth cameras address the privacy issues of visual/thermal camera solutions, however, have a limited field of view and require an unobscured side-profile view of their subjects, introducing problems with deployment. Vibration detection based solutions detect vibrations in a locale that may represent a fall but are subject to false positives. Positional tracking, incorporating technology such as pressure-sensitive floors or radar, are effective, however, expensive and require an extensive retrofitting of an environment. Thermal vision has been previously investigated using neural networks and the verticalvelocity of a person. This solution had issues related to low detection rate, limited field of view, requirement for an unobscured side-profile view and was easily confused by a person sitting down.

In order to address these shortcomings, a novel approach to detecting falls, related to the work in [25] has been proposed. This approach uses ceiling mounted thermal vision sensors in conjunction with an advanced computer vision based mechanism which identifies potential falls. Potential falls are logged with alerts being passed to caregiving staff who can then intervene appropriately. In this approach, an individual's environment is monitored in a way that is non-invasive and respects privacy.

\section{Detecting falls through thermal vision}

The devised fall detection approach observes an inhabitant through a thermal vision sensor and identifies likely fall events. Thermal sensors are placed on a ceiling and generate a $32 * 31$ frame of temperature readings for the area directly below at a rate of $6 \mathrm{~Hz}$. This sensor has an extremely wide field of view of $86^{\circ}$ by $83^{\circ}$, allowing perception of a large area; at a deployment of height of 2.5 meters the viewable areas is approximately 6 meters by 5.6 meters. This sensor is shown in Figure 1 (a) and its perception of the world is shown in Figure 1 (b). 


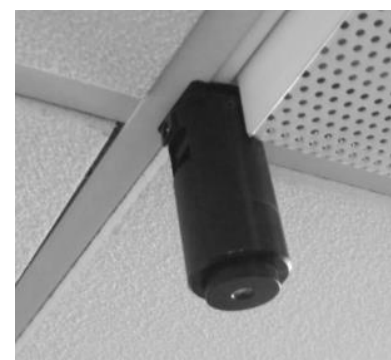

(a)

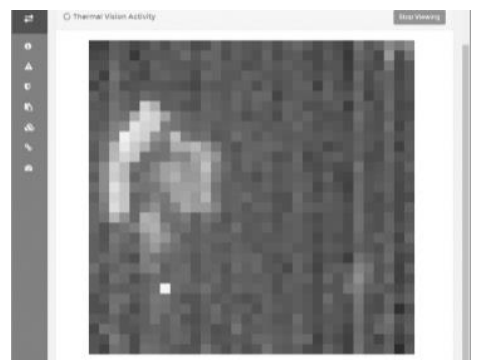

(b)

Fig. 1. The thermal vision sensor used in this study (a). The environment as perceived by the sensor and presented in a web interface, the hot readings are represented by white pixels (b).

Frames are read from the sensor through a listener program placed on the local network. This listener reads raw frame data from this sensor and interleaves it to generate frames with usable values in Celsius, to 2 decimal places. The listener then forwards these usable frames to a web service endpoint. This web service endpoint then stores the frame in a Time Series Database (TSDB) and an in-memory cache.

A computer vision process retrieves the frame data from the endpoint and performs blob and entity detection. Initially, the process filters thermal pixels which are outside of the likely range of human emissive temperatures or occurred through gradually raised temperature. These filtered readings are subsequently used to identify thermal pixels that are significantly above unfiltered background temperatures through comparison with previous frame data. These identified thermal pixels are then grouped as blobs, representing occupants, and a number of metrics are generated. These metrics include thermal pixel count, blob location, blob emissive temperature, blob width, blob height and blob aspect ratio. Finally, blobs that have a thermal pixel count less than 6 are discarded, as these would be too small to represent a human at the intended sensor deployment height, as determined though a small number of evaluations. Further information on this process can be obtained in [26, 27].

This blob detection process then stores these blob in the TSDB and memory cache. A Fall Detection Process (FDP) subsequently consumes these recorded blob ${ }^{1}$. If the FDP detects a fall, it is recorded in a Relational Database which is presented in a web interface. The overall architecture of this approach is presented in Figure 2 and is followed by a description of the FDP.

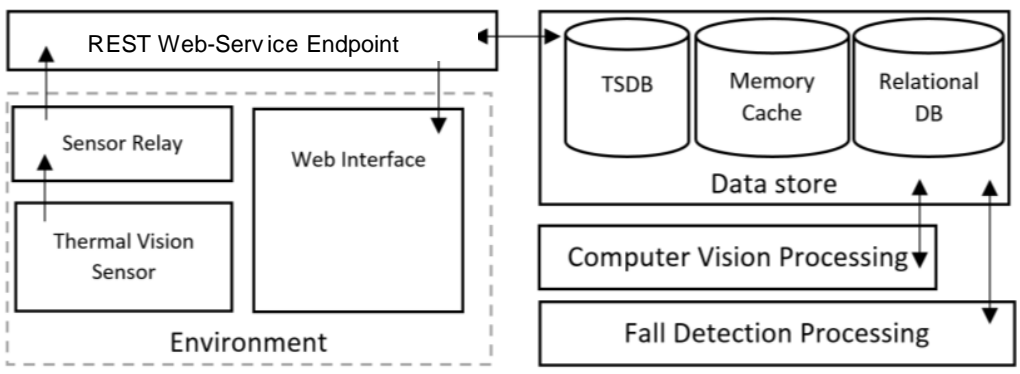

Fig. 2. The architecture of the developed fall detection platform.

1 It should be noted that this detection process is designed to operate within a single occupant environment, when a fall is most dangerous and there is no immediate assistance available. 
The FDP reasons upon the blob records generated by the computer vision process. Central to this process is the hypothesis that rapid and significant expansion of a blob in a localized area could indicate a fall.

Blobs under consideration are those within body temperate range, $26 \mathrm{C}-41 \mathrm{C}$. Only blobs that have fully entered the frame are considered, as the rapid blob expansion from an inhabitant entering a scene could mislead the FDP. Blobs are considered to be in scene when they are not touching the outer pixels of the frame. Although this reduces the usable area of sensor frames, uncertainty related to partial observability is reduced. Events in which multiple blobs converge into one, potentially representing close conversation, are ignored by the FDP to reduce false positives.

When a single, unconverged and in scene blob is present the FDP is eligible and so performed. The FDP operates on a window of 4 frames; where $f_{n}$ is the current frame and $f_{n-3}$ is the $4^{\text {th }}$ most current frame. A reference blob size value, $b l o b_{r s}$, is produced by averaging the size of a blob from $f_{n-3}$ and $f_{n-2}$. Averaging over 2 frames introduces a smoothing factor, reducing the impact of anomalous readings and accommodating movement. blob $b_{r s}$ is assumed to initially indicate the size of a blob in a non-fall state, such as standing. If at any point, the blob size in $f_{n}$ expands more than 1.8 times larger than $b l o b_{r s}$ it will be assumed that a fall has occured. Frame $f_{n-1}$ is not considered to facilitate transitioning from a normal posture to a fall. The value of 1.8 was arose through sequentialanalysis of values between 1.2 and 3.4 incremented in steps of 0.2.

This analysis incorporated 4 test cases, involving one subject; 2 test falls and 2 postural changes incorporating sitting to seating and vice versa. 1.8 was the lowest value to reliably detect test falls and not be triggered on posture transitions. Higher values may reduce false positives, however, not detect falls. If other blobs enter the scene, this process is reset and halted until its eligibility is restored. Following this initial development, this FDP was then evaluated; the scenario and results of which are presented in the following Section.

\section{Evaluation}

In order to evaluate the suitability of this approach it was necessary to evaluate fall detection rates and false positives. To achieve this, a sensor was deployed in a test area for two 4-hour evaluation periods. During these periods a test subject performed their daily tasks, occasionally interacted with other occupants, and performed two sets of simulated falls. Daily tasks included working at a computer, reading, and making coffee. During performance of daily tasks and interaction a manual record of fall events served as a ground truth during performance of the simulated falls. Thirty Postural Changes (PC) were performed to gain insight into false positives and false negatives. PCs are significant changes in blob size through normal activity, such as reaching across a desk or moving from seated to standing. The results of this evaluation are presented in Table 1.

Table 1. Accuracy of fall detection through the thermal vision-based FDP devised in this study.

\begin{tabular}{cccccccc}
\hline \multirow{2}{*}{ Set } & $\begin{array}{c}\text { Simulated } \\
\text { Falls }\end{array}$ & $\begin{array}{c}\text { Detected Detection } \\
\text { Falls }\end{array}$ & PC & PC & True Positive/False Positive/True & Sensitivity/ \\
& 15 & 10 & $66.7 \%$ & 20 & 4 & Negative/False Negative & Specificity \\
\hline I & 15 & 7 & $70 \%$ & 10 & 3 & $10 / 4 / 16 / 5$ & $66.7 \% / 80.0 \%$ \\
II & 10 & 7 & & $7 / 3 / 7 / 3$ & $70.0 \% / 70.0 \%$ \\
\hline
\end{tabular}


The preliminary results show that this approach has promise, though a number of is sues were highlighted. In some cases, PCs were incorrectly identified as falls. Incorrectly classified PCs included reaching across a desk with a leg extended for balance, which is not likely to be performed by an elderly person in the home, and quickly transitioning from standing to seated while arms were simultaneously being fully extended. Some falls were not correctly identified when the subject limbs where partially obscured by desks and other fixtures. In another case a slow fall was not detected where the subject partially stopped their fall by pushing against a support. Potential mechanisms to address these errors are presented in the conclusion of this paper.

\section{Conclusion}

In this paper we present a novel approach to fall detection that utilizes thermal vision sensors and a computer vision based FDP. This approach addresses some is sues with previous fall detection works by respecting privacy, not requiring an extensive retrofitting process and eliminating the need for wearable devices. Although this study is in its early stage, preliminary evaluation shows accuracy of $68 \%$, comparing favorably with previous thermal vision fall detection that was 35\% accurate [25].

A number of deficiencies have been identified and efforts are underway to address these. Specifically, edge and shape detection will be added to the computer vision process to reduce misclassification of unusual PCs. Additional work, will investigate use of shape detection to help address issues with falls where the subject becomes partially obscured. Finally, adaptive generation of blob $b_{r s}$ across a dynamic frame window will be investigated to accommodate variance in fall rates.

Following development of these improvements, a more thorough evaluation will be undertaken involving a number of subjects and a larger sample size. Following evaluation, trialing this approach in a suitable population will be investigated.

Acknowledgments. Invest Northern Ireland is acknowledged for supporting this project under the Competence Centre Programs Grant RD0513853 - Connected Health Innovation Centre.

\section{$6 \quad$ References}

1. Evans, D., Pester, J., Vera, L., Jeanmonod, D., Jeanmonod, R.: Elderly fall patients triaged to the trauma bay: Age, injury patterns, and mortality risk. Am. J. Emerg. Med. 33, 1635 1638 (2015).

2. González, N., Aguirre, U., Orive, M., Zabala, J., García-Gutiérrez, S., Las Hayas, C., Navarro, G., Quintana, J.M.: Health-related quality of life and functionality in elderly men and women before and after a fall-related wrist fracture. Int. J. Clin. Pract. 68, 919-928 (2014).

3. Stevens, J. a, Corso, P.S., Finkelstein, E. a, Miller, T.R.: The costs of fatal and non-fatal falls among older adults. Inj. Prev. 12, 290-295 (2006).

4. Masud, T., Morris, R.O.: Epidemiology of falls. Age Ageing. 30, 3-7 (2001).

5. El-Khoury, F., Cassou, B., Charles, M.-A., Dargent-Molina, P.: The effect of fall prevention exercise programmes on fall induced injuries in community dwelling older adults: systematic review and meta-analysis of randomised controlled trials. Bmj. 347 , f6234 (2013).

6. Coppedge, N.: Using a standardized fall prevention tool decreases fall rates. Nursing (Lond). 46, 64-67 4p (2016).

7. Lord, S.R., Sherrington, C., Menz, H.B., Close, J.C.T.: Falls in older people: risk factors and strategies for prevention. Cambridge University Press (2007). 
Bagal, F., Becker, C., Cappello, A., Chiari, L., Aminian, K., Hausdorff, J.M., Zijlstra, W., Klenk, J.: Evaluation of accelerometer-based fall detection algorithms on real-world falls. PLoS One. 7, 1-9 (2012).

9. Khan, S.S., Hoey, J.: Review of Fall Detection Techniques: A Data Availability Perspective. (2016).

10. Zhang, Z., Conly, C., Athitsos, V.: A Survey on Vision-based Fall Detection. Proc. 8th ACM Int. Conf. PErvasive Technol. Relat. to Assist. Environ. 46:1-46:7 (2015).

11. Schwickert, L., Becker, C., Lindemann, U., Maréchal, C., Bourke, A., Chiari, L., Helbostad, J.L., Zijlstra, W., Aminian, K., Todd, C., others, Group, C.: Fall detection with body -worn sensors. Z. Gerontol. Geriatr. 46, 706-719 (2013).

12. Pannurat, N., Thiemjarus, S., Nantajeewarawat, E.: Automatic fall monit oring: a review. Sensors (Basel). 14, 12900-12936 (2014).

13. Ciuti, G., Ricotti, L., Menciassi, A., Dario, P.: MEMS sensor technologies for human centred applications in healthcare, physical activities, safety and environmental sen sing: A review on research activities in Italy. Sensors. 15, 6441-6468 (2015).

14. Tsai, P., Yang, Y., Shih, Y., Kung, H.: Gesture-Aware Fall Detection System : Design and Implementation. 88-92 (2015).

15. Lee, J.K., Robinovitch, S.N., Park, E.J.: Inertial Sensing-Based Pre-Impact Detection of Falls Involving Near-Fall Scenarios. IEEE Trans. Neural Syst. Rehabil. Eng. 23, 258-266 (2015).

16. Rakhman, A.Z., Nugroho, L.E., Widyawan, Kurnianingsih: Fall detection system using accelerometer and gyroscope based on smartphone. 2014 st Int. Conf. Inf. Technol. Comput. Electr. Eng. Green Technol. Its Appl. a Better Futur. ICIT ACEE 2014 - Proc. 99 104 (2015).

17. Aguiar, B., Rocha, T., Silva, J., Sousa, I.: Accelerometer-based fall detection for smartphones. In: IEEE MeMeA 2014 - IEEE International Symposium on Medical Measurements and Applications, Proceedings (2014).

18. Salman Khan, M., Yu, M., Feng, P., Wang, L., Chambers, J.: An unsupervised acoustic fall detection system using source separation for sound interference suppression. Signal Processing. 110, 199-210 (2015).

19. Sokolova, M. V., Serrano-Cuerda, J., Castillo, J.C., Fernndez-Caballero, A.: A fuzzy model for human fall detection in infrared video. J. Intell. Fuzzy Syst. 24, 215-228 (2013).

20. Debard, G., Baldewijns, G., Goedem, T., Tuytelaars, T., Vanrumste, B.: Camera-based fall detection using a particle filter. 6947-6950(2015).

21. Zigel, Y., Litvak, D., Gannot, I.: A method for automatic fall detection of elderly people using floor vibrations and soundProof of concept on human mimicking doll falls. IEEE Trans. Biomed. Eng. 56, 2858-2867 (2009).

22. Chaccour, K., Darazi, R.: Smart Carpet using differential piezoresistive pressure sensors for elderly fall detection. Presented at the (2015).

23. Amin, M.G., Zhang, Y.D., Ahmad, F., Ho, K.C.D.: Radar signal processing for elderly fall detection: The fut ure for in-home monitoring. IEEE Signal Process. Mag. 33, 71-80 (2016).

24. Loncomilla, P., Tapia, C., Daud, O., Ruiz-del-Solar, J.: A Novel Methodology for Assessing the Fall Risk Using Low-Cost and Off-the-Shelf Devices. IEEE Trans. HumanMachine Syst. 44, 406-415 (2014).

25. Sixsmith, A., Johnson, N.: A smart sensor to detect the falls of the elderly. IEEE Pervasive Comput. 3, (2004).

26. Rafferty, J., Synnott, J., Nugent, C.: A Hybrid Rule and Machine Learning Based Generic Alerting Platform for Smart Environments. Engineering in Medicine and Biology Society. In: Engineering in Medicine and Biology Society (EMBC), 2016 38th Annual International Conference of the IEEE (2016).

27. Synnott, J., Nugent, C., Jeffers, P.: A Thermal Data Simulation Tool for the Testing of Novel Approaches to Activity Recognition. In: Pecchia, L., Chen, L., Nugent, C., and Bravo, J. (eds.) Ambient Assisted Living and Daily Activities SE - 2. pp. 10-13. Springer International Publishing (2014). 